\title{
Simple solutions of relativistic hydrodynamics for systems with ellipsoidal symmetry
}

\author{
T. Csörgő, ${ }^{\text {a,c,1 }}$ L. P. Csernai ${ }^{\mathrm{b}, \mathrm{a}, 2}$ Y. Hama, ${ }^{\mathrm{c}, 3}$ T. Kodama ${ }^{\mathrm{d}, 4}$ \\ a MTA KFKI RMKI, H - 1525 Budapest 114, POBox 49, Hungary \\ ${ }^{\mathrm{b}}$ Section for Theoretical Physics, Department of Physics, \\ University of Bergen, Allegaten 55, 5007 Bergen, Norway \\ ${ }^{\mathrm{c}} I F$ - USP, C.P. 66318, 05389-970 São Paulo - SP, Brazil \\ ${ }^{\mathrm{d}}$ IF - UFRJ, C.P. 68528, 21945-970 Rio de Janeiro - RJ, Brazil
}

\begin{abstract}
Simple, self-similar, analytic solutions of $(1+3)$-dimensional relativistic hydrodynamics are presented for ellipsoidally symmetric finite fireballs corresponding to non-central collisions of heavy ions at relativistic bombarding energies. The hydrodynamical solutions are obtained for a new, general family of equations of state with the possibility of describing phase transitions.
\end{abstract}

Key words: Relativistic hydrodynamics, ellipsoidal symmetry, equation of state, flow, analytic solutions

\section{Introduction}

Recently, new families of exact analytic solutions of relativistic hydrodynamics have been found in a Hungarian-Brazilian collaboration. The simplest case corresponds to the (1+1)-dimensional expansions [1]: it consists of a class of 1-dimensional scaling flows with a proper-time dependent pressure, but characterized with an arbitrary rapidity distribution and a temperature field that is coupled to the rapidity distribution, thus overcoming one of the shortcomings of the well-known Hwa-Bjorken solution [2], which has a flat rapidity distribution. Physically, the situation described in [1] may characterize e.g. soft

\footnotetext{
Email: csorgo@sunserv.kfki.hu

$2 \quad$ csernai@fi.uib.no

$3 \quad$ hama@fma.if.usp.br

$4 \quad$ tkodama@if.ufrj.br
} 
collisions of hadrons at high bombarding energies. These solutions are obtained for a broad class of equations of state that may include massive particles and an arbitrary constant of proportionality between the kinetic energy density and the pressure. The $(1+1)$-dimensional solutions have been generalized to the case of cylindrical symmetry in ref. [3], describing a physical situation that may correspond to central collisions of heavy ions at ultra-relativistic colliding energies, thus, overcoming another shortcoming of Hwa-Bjorken solution, which contains no transverse flow. These exact analytic solutions, reported in refs. $[1,3]$, are associated to each non-negative scaling function $\mathcal{V}(s)$ that satisfies the normalization condition $\mathcal{V}(0)=1$, where the argument $s$ is a scaling variable that guarantees the self-similarity of the solutions. Here we present a broader class of solutions belonging to this new family of exact solutions. In particular, we shall describe two classes of ellipsoidally symmetric solutions of relativistic hydrodynamics, that may be relevant for the study of non-central collisions of heavy ions at relativistic bombarding energies.

In general, exact analytic resolution of 3 dimensional relativistic hydrodynamics is a difficult task due to the highly non-linear nature of these equations. The complications are sometimes simplified by assuming idealized boundary conditions and simplified equations of state (e.g. infinite bombarding energy and a massless relativistic gas in the case of Hwa-Bjorken's solution [6]). A more realistic but analytically more complicated solution had been found by Khalatnikov [4], following Landau's basic ideas [5], that gave rise to the hydrodynamical approach in high-energy physics. Both of these solutions are frequently utilized as the basis for estimations of various observables in ultrarelativistic nucleus-nucleus collisions [6].

Analytic solutions of relativistic hydrodynamics were also reported recently in refs. $[7,8]$. However, these solutions are valid only at the softest point of the equation of state. For more realistic situations, the $(1+3)$-dimensional relativistic hydrodynamical equations are frequently solved using various numerical methods, for example, recent solutions were obtained with the help of smoothed-particle hydrodynamics in refs. [9,10].

The exact analytic solutions, reported here, are generalizations of the results of refs. $[1,3]$ to the case of 3 -dimensional relativistic expansions with less and less symmetry. However, we emphasize that these results also correspond to generalizations of earlier analytic solutions of non-relativistic hydrodynamics. The first solutions of this kind have been found by Zimányi, Bondorf and Garpman (ZBG) in 1978 [11]. The key aspect of the ZBG solution is its self-similarity, this property is kept in all the subsequent generalizations including cylindrical or ellipsoidal symmetries and relativistic flows. The ZBG solution has been generalized to the case of spheroidally symmetric expansions in ref. [12], to spherically symmetric Gaussian expansions in ref. [13]. From the point of view of the relativistic generalizations, an important stepping stone was made 
in ref. [14] when a scaling function has been introduced for the spherically symmetric non-relativistic solutions of hydrodynamics. This was the first case when a whole new family of solutions has been found, that assigned an independent solution for every (differentiable and integrable) scaling function that satisfied the requirement of positivity $\mathcal{T}(s)>0$ and normalized as $\mathcal{T}(0)=1$. The ZBG and the spherical Gaussian solutions appeared as special choices for the functional form of the scaling function $\mathcal{T}(s)$. The Gaussian family of spherical solutions has been generalized to Gaussian ellipsoidal expansions in refs. $[15,16]$. Recently, the whole family of self-similar ellipsoidally symmetric non-relativisitic solutions of hydrodynamics has been found in ref. [17]. In the present paper, we report on the generalization of this family of solutions to the case of relativistic expansions with ellipsoidal symmetry and introduce new families of relativistic solutions with even less than ellipsoidal symmetry. All the solutions reported here have non-trivial non-relativistic limiting behaviour.

\section{The equations of relativistic hydrodynamics.}

Let us adopt the following notational conventions: the coordinates are $x^{\mu}=$ $(t, \mathbf{r})=\left(t, r_{x}, r_{y}, r_{z}\right), x_{\mu}=\left(t,-r_{x},-r_{y},-r_{z}\right)$ and the metric tensor is $g^{\mu \nu}=$ $g_{\mu \nu}=\operatorname{diag}(1,-1,-1,-1)$. The relativistic continuity and energy-momentumconservation equations are

$$
\begin{aligned}
\partial_{\mu}\left(n u^{\mu}\right) & =0, \\
\partial_{\nu} T^{\mu \nu} & =0,
\end{aligned}
$$

where $n \equiv n(x)$ is the conserved number density, $u^{\mu} \equiv u^{\mu}(x)=\gamma(1, \mathbf{v})$ is the four-velocity, with $u^{\mu} u_{\mu}=1$, and $T^{\mu \nu}$ is the energy-momentum tensor. We assume perfect fluid,

$$
T^{\mu \nu}=(\epsilon+p) u^{\mu} u^{\nu}-p g^{\mu \nu},
$$

where $\epsilon$ stands for the relativistic energy density and $p$ is the pressure.

From general thermodynamical considerations and the perfectness of the fluid one can show that the expansion is adiabatic,

$$
\partial_{\mu}\left(\sigma u^{\mu}\right)=0,
$$

where $\sigma$ stands for the entrophy density.

For the equations of state, we assume a gas that may contain massive conserved 
quanta,

$$
\begin{aligned}
& \epsilon=m n+\kappa p, \\
& p=n T .
\end{aligned}
$$

These equations have two free parameters, $m$ and $\kappa$. Non-relativistic hydrodynamics of ideal gases corresponds to the limiting case of $\mathbf{v}^{2} \ll 1$ and $\kappa=\frac{3}{2}$. Our solutions, presented below, exist for all values of $m \geq 0$ and $\kappa>0$.

Using the continuity equation (1) and the equations of state $(5,6)$, the energymomentum-conservation equations, (2) can be transformed to the Euler and temperature equations,

$$
\begin{gathered}
u_{\nu} u^{\mu} \partial_{\mu} p+(\epsilon+p) u^{\mu} \partial_{\mu} u_{\nu}-\partial_{\nu} p=0 \\
u^{\mu} \partial_{\mu} T+\frac{1}{\kappa} T \partial_{\mu} u^{\mu}=0
\end{gathered}
$$

Expressing the energy density and the pressure in the 3 independent components of the relativistic Euler equation in terms of $n$ and $T$ with the help of the equations of state, $(5,6)$, one obtains a closed system of 5 equations (the continuity, the Euler and the temperature equations) in terms of 5 variables, $n, T$ and $\mathbf{v}=\left(v_{x}, v_{y}, v_{z}\right)$.

\section{Self-similar, ellipsoidally symmetric ansatz}

We search for self-similar solutions in which the isotherms at each instant are ellipsoidal surfaces, where the number density is also constant. We do not discuss here how the major axes of these ellipsoids may be rotated in the frame of observation. We describe the solution in the natural System of Ellipsoidal Expansion (SEE) $[15,16]$, where the coordinate axes point to the pricipal directions of the expansion. Let us now define the following scaling variable

$$
s=\frac{r_{x}^{2}}{X^{2}}+\frac{r_{y}^{2}}{Y^{2}}+\frac{r_{z}^{2}}{Z^{2}}
$$

where the scale parameters $X=X(t), Y=Y(t)$ and $Z=Z(t)$ are assumed to depend only on the time variable $t$. The condition $s=$ const., at each instant $t$, defines the isotherms mentioned above. As these surfaces are ellipsoids, the solutions belonging to this class will be characterized by ellipsoidal symmetry. We assume a self-similar expansion of Hubble type, with possibly different 
Hubble constants in all the pricipal directions of the expansion:

$$
\begin{aligned}
& v_{x}(t, \mathbf{r})=\frac{\dot{X}}{X} r_{x} \\
& v_{y}(t, \mathbf{r})=\frac{\dot{Y}}{Y} r_{y} \\
& v_{z}(t, \mathbf{r})=\frac{\dot{Z}}{Z} r_{z}
\end{aligned}
$$

where $\dot{A}=d A(t) / d t$ stands for the derivative of the scale parameter $A=$ $\{X, Y, Z\}$ with respect to time $t$.

As a straightforward generalization of the results derived in refs $[1,3]$, we find the following new family of ellipsoidally symmetric, exact analytic solutions of relativistic hydrodynamics:

$$
\begin{array}{rlrl}
s & =\frac{r_{x}^{2}}{\dot{X}_{0}^{2} t^{2}}+\frac{r_{y}^{2}}{\dot{Y}_{0}^{2} t^{2}}+\frac{r_{z}^{2}}{\dot{Z}_{0}^{2} t^{2}}, & \\
\mathbf{v} & =\frac{\mathbf{r}}{t} \quad \text { or } \quad u^{\mu}=\frac{x^{\mu}}{\tau} \\
n(t, \mathbf{r}) & =n_{0}\left(\frac{\tau_{0}}{\tau}\right)^{3} \mathcal{V}(s), \\
p(t, \mathbf{r}) & =p_{0}\left(\frac{\tau_{0}}{\tau}\right)^{3+3 / \kappa}, \\
T(t, \mathbf{r}) & =T_{0}\left(\frac{\tau_{0}}{\tau}\right)^{3 / \kappa} \frac{1}{\mathcal{V}(s)}
\end{array}
$$

where $\tau=\sqrt{t^{2}-\mathbf{r}^{2}}, p_{0}=n_{0} T_{0}, \dot{X}_{0}, \dot{Y}_{0}, \dot{Z}_{0}$ are constants and $\mathcal{V}(s)$ is an arbitrary positive, differentiable function of the scaling variable $s$ normalized such that $\mathcal{V}(0)=1$. The temperature distribution also depends on the scaling variable $s$ through a scaling function $\mathcal{T}(s)$, which happens to be $\mathcal{T}(s)=$ $1 / \mathcal{V}(s)$. Note that the pressure $p$ depends only on $\tau$.

An interesting property of this solution is that the flow and the pressure fields are spherically symmetric, however, the other thermodynamical quantities such as the temperature or density distributions are characterized by ellipsoidal symmetry.

The lack of acceleration is reflected by the equations

$$
\begin{aligned}
& X=\dot{X}_{0} t, \\
& Y=\dot{Y}_{0} t \\
& Z=\dot{Z}_{0} t
\end{aligned}
$$

In this family of new solutions, the flow is three-dimensional, accelerationless 
Hubble-type, $u^{\mu}=x^{\mu} / \tau$, which gives

$$
u^{\mu} \partial_{\mu} u_{\nu}=0
$$

This property, together with the $s$-independence of pressure fields, guarantees that the Euler equation be satisfied regardless of the mass $m$ and the value of $\kappa$. We have found new, self-similar, scaling solutions to the continuity and the temperature equations with ellipsoidal symmetry. Thus a new hydrodynamical solution is assigned to each scaling function $\mathcal{V}(s)=1 / \mathcal{T}(s)$, similarly to the cases of the ellipsoidally symmetric, non-relativistic solutions of ref. [17].

\section{Phase transitions and freeze-out}

The simplest case of the previously discussed new family of exact solutions of relativistic hydrodynamics is given by the choice of $\mathcal{V}(s)=1$. In this case, the temperature is constant on a hypersurface characterized by a constant proper-time, and all the other thermodynamical parameters are constants on these surfaces as well. Thus these parameters depend on $\tau$ only, similarly to the Hwa-Bjorken solution [2].

It is possible to generalize the equations of state, $(5,6)$ to characterize a new phase of matter and then describe the rehadronization of a "Quark Matter" (QM) state to a Hadron gas (H) in a model similar to that of Gyulassy and Matsui [18]. For simplicity, from now on we refer to the the equations of state $(5,6)$, describing massive, conserved quanta, as that of a hadron gas and index the variables with subscript $H$. To phenomenologically describe a new phase, which includes constituent quarks and anti-quarks (Q), characterized by their mass and by a vacuum pressure, we generalize the equations of state to

$$
\begin{aligned}
& \varepsilon_{Q}=m_{Q} n_{Q}+\lambda_{\varepsilon} n_{Q} T+B, \\
& p_{Q}=\lambda_{p} n_{Q} T-B
\end{aligned}
$$

where $\lambda_{\varepsilon}$ and $\lambda_{p}$ are constants. Let us introduce the notation $\kappa_{Q}=\lambda_{\varepsilon} / \lambda_{p}$. With this simple ansatz, the form of the relativistic Euler and temperature equations does not change and we obtain the same form of the solutions for $n$, $T$ and $\mathbf{v}$ as before, however, with modified boundary conditions corresponding to modified constants of integration. We may refer to this new phase as a kind of quark matter, providing simple model equations of state for massive quarks, and a bag constant corresponding to color deconfinement. The gluons are assumed to be integrated out, providing mass for the quarks. Such a picture is qualitatively supported by phenomenological fits to the lattice QCD equation

of state with quarks that pick up the constitutent mass around $T_{c}$ and gluons that carry big effective mass in the same temperature domain [19]. 
Using the above ansatz for the equations of state, we obtain the following solution for the pressure:

$$
p_{Q}=\left(p_{0, Q}+B\right)\left(\frac{\tau_{0}}{\tau}\right)^{3+3 / \kappa_{Q}}-B
$$

If we chose $B>0$ and $\lambda_{p}>1$, then at low temperatures the state consisting of non-relativistic massive ideal gas will be stable, and at some critical temperature $T_{c}$ the pressure of the two phases is balanced. However, at high temperatures, the pressure of the new phase will be larger than that of the hadronic matter, as $\lambda_{p}>1$, hence at high temperatures this phase will be the stable one.

The critical temperature, $T_{c}$ is defined by

$$
n_{H} T_{c}=\lambda_{p} n_{Q} T_{c}-B
$$

For the hydrodynamical description, the specification of $n_{H}$ and $n_{Q}$ as a function of e.g. a temperature and chemical potential is not necessary, any thermodinamically consistent form can be included into the hydro solutions that we focus on. Hence the above equation may indicate, if $n_{H}$ and $n_{Q}$ are expressed as a function of the temperature and the (baryo)chemical potential, that the critical temperature may become density dependent.

One can show that the expansion is adiabatic if the matter consists of only one of the phases. We may assume, that the expansion is adiabatic also during the period of phase coexistence, and describe the phase transition with the help of a Maxwell construction. This case is similar to the construction discussed by Gyulassy and Matsui in case of a massless ideal gas [18]. Let us denote the entrophy density of the two phases by $s_{Q}$ and $s_{H}$, and the volume fraction of the two phases by $f_{Q}$ and $f_{H}=1-f_{Q}$, respectively. The proper-time dependence of the entrophy density is then given by

$$
s(\tau)=f_{Q}(\tau) s_{Q, c}+\left(1-f_{Q}(\tau)\right) s_{H, c} .
$$

At the beginning of the phase transition, at $\tau=\tau_{Q, c}$ the whole system consists of "Quark Matter", that is $f_{Q}\left(\tau_{Q, c}\right)=1$. By the time the phase transition ends, one obtains $f_{Q}\left(\tau_{H, c}\right)=0$. As the well known steps of the Maxwell construction can be copied from the case without conserved charges, for further details we simply refer to section 6.2 .3 of ref. [6].

Starting the hydrodynamical expansion from a quark matter initial state, the form of our hydrodynamical solution becomes unmodified even during the phase transition, and the coasting type of hydrodynamical evolution can be continued at a fixed $T_{c}$, from $\tau_{Q, c}$ to $\tau_{H, c}$ untill all matter is transformed to hadronic matter. Then one can continue the same kind of coasting solution untill the hadronic matter freezes out. 
This expansion takes usually a long time, which is difficult to justify by experimental evidences. However, the same kind of hydrodynamical solution can be matched with a non-equilibrium type of phase transition, the sudden rehadronization and a simultaneous freeze-out of hadrons from a supercooled Quark Matter, as described in greater details in refs. [20,21]. Then in the same model one may reach a deeply supercooled Quark Matter state, and the transition to the hadron gas may proceed via a mechanical instability, associated with a negative pressure state, governed by the conservation of matter, energy and momentum and the impossibility of an entrophy decrease in a deflagration through a hypersurface with a time-like normal vector at $\tau=\tau_{T D}$,

$$
\begin{aligned}
& {\left[T^{\mu \nu} d f n_{\nu}\right]=0} \\
& {\left[n u^{\nu} d f n_{\nu}\right]=0} \\
& {\left[s u^{\nu} d f n_{\nu}\right] \geq 0}
\end{aligned}
$$

where $d f n_{\nu} \equiv d \sigma_{\nu}$ is the normal-vector of the $\tau=\tau_{T D}$ hypersurface. These equations and inequality can be solved very similarly to ref. [20], that discussed the case of a transition from a massless ideal quark-gluon plasma (QGP) to a massless hadron gas. The lack of acceleration, and the invariance of the equations of state for rescaling the temperature and for adding a vacuum energy term are the essential reasons why earlier considerations that discussed phase transitions for massless particles can be straigthforwardly implemented to our new family of exact hydrodynamical solutions that describe the phase transitions and expansions of massive quanta.

Recently, a new method has been proposed to evaluate particle emission from hydrodynamically evolving, locally thermalized sources, that generalizes the Cooper-Frye freeze-out conditions for systems with volume emission [23,22]. This method, based on the method of escaping probabilities, can also be applied to evaluate the particle spectra and correlation functions from the new hydrodynamical solutions, as the non-relativistic form of the solutions corresponds to the limiting behaviour that was discussed in ref. [23].

\section{$5 \quad$ Factorized solutions}

From the structure of the new family of solutions presented above, it is obvious how to generate further new solutions of relativistic hydrodynamics.

Observe that a modified version of the scaling variable of eq. (13) can be defined, and another family of the same kind of ellipsoidal solutions can be generated by introducing 


$$
\begin{aligned}
s^{\prime} & =\frac{r_{x}^{2}}{\dot{X}_{0}^{2} \tau^{2}}+\frac{r_{y}^{2}}{\dot{Y}_{0}^{2} \tau^{2}}+\frac{r_{z}^{2}}{\dot{Z}_{0}^{2} \tau^{2}}, \\
X^{\prime} & =\dot{X}_{0} \tau \\
Y^{\prime} & =\dot{Y}_{0} \tau \\
Z^{\prime} & =\dot{Z}_{0} \tau
\end{aligned}
$$

where the dots should be understood as derivatives with respect to $\tau$. Mathematically, the modified scaling variable solves the relativistic hydro equations, because it also satisfies the requirement

$$
u^{\mu} \partial_{\mu} s=u^{\mu} \partial_{\mu} s^{\prime}=0
$$

if the flow is scaling. This condition can be considered also as the criterium of a "good" scaling variable, and satisfied by the following independent sets of variables:

$$
\begin{aligned}
& s_{x}=\frac{r_{x}^{2}}{t^{2}}, \\
& s_{y}=\frac{r_{y}^{2}}{t^{2}}, \\
& s_{z}=\frac{r_{z}^{2}}{t^{2}},
\end{aligned}
$$

or alternatively,

$$
\begin{aligned}
& s_{x}^{\prime}=\frac{r_{x}^{2}}{\tau^{2}} \\
& s_{y}^{\prime}=\frac{r_{y}^{2}}{\tau^{2}} \\
& s_{z}^{\prime}=\frac{r_{z}^{2}}{\tau^{2}} .
\end{aligned}
$$

All of the 6 scale variables defined above satisfy eq. (34) if the flow profile is of a 3-dimensional scaling type, $u^{\mu}=x^{\mu} / \tau$.

As the relationship $\mathcal{V}(s)=1 / \mathcal{T}(s)$ holds in the class of solutions we present here, if we assume a factorized form for the scaling function of the density, we automatically generate a factorized form for the scaling function of the temperature, hence it becomes easy to generate further new solutions. Let us define the scaling functions $\mathcal{V}_{x}\left(s_{x}\right), \mathcal{V}_{y}\left(s_{y}\right)$ and $\mathcal{V}_{z}\left(s_{z}\right)$ that are positive, differentiable and satisfying $\mathcal{V}_{x}(0)=\mathcal{V}_{y}(0)=\mathcal{V}_{z}(0)=1$, otherwise being abritrary 
and independent from each other. Then, a new type of hydro solutions reads

$$
\begin{array}{rlrl}
\mathbf{v} & =\frac{\mathbf{r}}{t} \quad \text { or } & u^{\mu}=\frac{x^{\mu}}{\tau} \\
n(t, \mathbf{r}) & =n_{0}\left(\frac{\tau_{0}}{\tau}\right)^{3} \mathcal{V}_{x}\left(s_{x}\right) \mathcal{V}_{y}\left(s_{y}\right) \mathcal{V}_{z}\left(s_{z}\right) \\
(t, \mathbf{r}) & =p_{0}\left(\frac{\tau_{0}}{\tau}\right)^{3+3 / \kappa}, \\
T(t, \mathbf{r}) & =T_{0}\left(\frac{\tau_{0}}{\tau}\right)^{3 / \kappa} \frac{1}{\mathcal{V}_{x}\left(s_{x}\right)} \frac{1}{\mathcal{V}_{y}\left(s_{y}\right)} \frac{1}{\mathcal{V}_{z}\left(s_{z}\right)}
\end{array}
$$

Note that this form of solution is invariant for a change of the scaling variables and the scales as $\left(s_{x}, s_{y}, s_{z}\right) \rightarrow\left(s_{x}^{\prime}, s_{y}^{\prime}, s_{z}^{\prime}\right)$ and $(X, Y, Z) \rightarrow\left(X^{\prime}, Y^{\prime}, Z^{\prime}\right)$, with the time derivatives in the definitions of the Hubble flow field, eqs. (10-12) understood as derivations with respect to $\tau$ as in eqs. (30-33).

\section{General form of solutions, beyond ellipsoidal symmetry}

The key point in checking that the above factorized forms indeed solve the equations of relativistic hydrodynamics is that the comoving derivatives of the scaling functions, $u_{\mu} \partial^{\mu} \mathcal{V}_{i}\left(s_{i}\right)$, are proportional to $u^{\mu} \partial_{\mu} s_{i}$, hence vanish. This is, together with $u^{\mu} \partial_{\mu} u_{\nu}=0$, the essential property of the scaling solutions.

We find that the most general form of the scaling variable is

$$
\bar{s}=F\left(s_{x}, s_{y}, s_{z}\right) \equiv G\left(s_{x}^{\prime}, s_{y}^{\prime}, s_{z}^{\prime}\right)
$$

which means that any function of scaling variables $\left(s_{x}, s_{y}, s_{z}\right)$ or $\left(s_{x}^{\prime}, s_{y}^{\prime}, s_{z}^{\prime}\right)$ can be utilized as a new scaling variable. Indeed, we have

$$
u_{\mu} \partial^{\mu} F\left(s_{x}, s_{y}, s_{z}\right)=\sum_{i=x, y, z} \frac{\partial F}{\partial s_{i}} u_{\mu} \partial^{\mu} s_{i}=0 .
$$

which yields the generalized form of the new family of solutions of relativistic hydrodynamics:

$$
\begin{array}{rlrl}
\mathbf{v} & =\frac{\mathbf{r}}{t} \quad \text { or } & u^{\mu}=\frac{x^{\mu}}{\tau} \\
n(t, \mathbf{r}) & =n_{0}\left(\frac{\tau_{0}}{\tau}\right)^{3} \mathcal{V}(\bar{s}), & \\
p(t, \mathbf{r}) & =p_{0}\left(\frac{\tau_{0}}{\tau}\right)^{3+3 / \kappa}, \\
T(t, \mathbf{r}) & =T_{0}\left(\frac{\tau_{0}}{\tau}\right)^{3 / \kappa} \frac{1}{\mathcal{V}(\bar{s})},
\end{array}
$$


with the constraint that $\mathcal{V}(0)=1$. For example, if we choose $\bar{s}=s_{x}+s_{y}+$ $s_{z}$, we obtain solutions with ellipsoidal symmetry, however, one may choose $\bar{s}=s_{x}+s_{y}-s_{z}$ to obtain solutions where the isotherms are one-sheeted hyperboloids, or $\bar{s}=-s_{x}-s_{y}+s_{z}$ where the temperature and the density are constants on two-sheeted hyperboloids. In fact the possibilities are infinitely rich.

\section{Non-relativistic limiting behaviour}

As the considered equation of state contains mass as a free parameter, it is possible to study the non-relativistic limiting case of these new relativistic solutions. The local thermal motion is non-relativistic if $m \gg T$, the flow is non-relativistic in the region of $|\mathbf{r}| \ll t$, which implies $\tau \approx t$ and relates the relativistic solutions with ellipsoidal symmetry to the asymptotic, large time behaviour of the ellipsoidally symmetric, non-relativistic solutions of refs. $[15,16,17]$. Let us recapitulate here the general form of the non-relativistic solutions of fireball hydrodynamics [17].

The definition of the scaling variable $s$ coincides with that of eq. (9). The solution for the flow field coincides with that of eqs. (10-12). The scaling function for the temperature, $\mathcal{T}(s)$ is utilized to express the solution for the NR density and temperature fields as

$$
\begin{aligned}
& n=n_{0} \frac{X_{0} Y_{0} Z_{0}}{X Y Z} \frac{1}{\mathcal{T}(s)} \exp \left[-\frac{m\left(\dot{X}_{\mathrm{as}}^{2}+\dot{Y}_{\mathrm{as}}^{2}+\dot{Z}_{\mathrm{as}}^{2}\right)}{T_{0}} \int_{0}^{s} d u \frac{1}{\mathcal{T}(u)}\right] \\
& T=T_{0}\left(\frac{X_{0} Y_{0} Z_{0}}{X Y Z}\right)^{1 / \kappa} \mathcal{T}(s)
\end{aligned}
$$

The large time behaviour of these non-relativistic, self-similar, ellipsoidal solutions of hydrodynamics is characterized by constant values of ( $\dot{X}_{\text {as }}, \dot{Y}_{\text {as }}, \dot{Z}_{\text {as }}$ ), which implies that for asymptotically long times a scaling flow field develops also in these non-relativistic solutions, $\mathbf{v} \approx \mathbf{r} / t$. In the exponential factor that appears in this non-relativisitic solution for $n$ we may expand the inverse scaling function $1 / \mathcal{T}(u)$ as a polinomial in $u$. Keeping the leading order terms, and performing the integration, we find that the exponential factor will be approximately a Gaussian factor. However, the widths of the Gaussians in all the directions will be proportional to the time. As we have assumed that $|\mathbf{r}| \ll t$, this exponential factor yields a factor of $1+\mathcal{O}\left(\mathbf{r}^{2} / t^{2}\right)$, hence we find the general asymptotic behaviour of the non-relativistic solutions to be the same form as the general solution of the relativistic solution of the hydrodynamical 
equations:

$$
\begin{aligned}
& n_{\mathrm{as}} \approx n_{\mathrm{as}} \frac{t_{0}^{3}}{t^{3}} \frac{1}{\mathcal{T}(s)} \\
& T_{\mathrm{as}} \approx T_{\mathrm{as}}\left(\frac{t_{0}}{t}\right)^{3 / \kappa} \mathcal{T}(s) .
\end{aligned}
$$

Due to the rescaling, the constants of normalization $n_{\text {as }}$ and $T_{\text {as }}$ are different from $n_{0}$ and $T_{0}$.

\section{Summary}

We have generalized the recently found new family of solutions of relativistic hydrodynamics to the case of expanding fireballs with ellipsoidal symmetry. The solutions contain an arbitrary scaling function $\mathcal{V}(s)$, restricted only by non-negativity and by the requirement of $\mathcal{V}(0)=1$, a very rich set of possible scaling variabes $s$, and 5 important parameters, the mass $m$, the parameter

$\kappa$ of the equation of state, the scale parameters $\dot{X}_{0}, \dot{Y}_{0}$ and $\dot{Z}_{0}$. Furthermore, we generalized the equations of state to describe a phase transition from a deconfined state consisting of massive (constituent) quarks and antiquarks to a state consisting of massive hadrons. Based on the scaling properties of the equations of state, as well as on the coasting nature of the expansion, we have shown that both the usual adiabatic Maxwell construction as well as the fast, non-equilibrium time-like deflagrations can be constructed and described within the considered class of hydrodynamical solutions.

We expect that some of these solutions of relativistic hydrodynamics may have future applications in non-central collisions of heavy ions at relativistic bombarding energies, a topic of great current research interest.

\section{Acknowledgments}

T. Cs. would like to Y. Hama and G. Krein for kind hospitality during his stay at USP and IFT, Sao Paulo, Brazil, and M. Gyulassy for his kind hospitality at Columbia University, New York. This work has been supported by a NATO Science Fellowship (Cs.T.), the OTKA grants T026435, T029158 and T034269 of Hungary, the NWO - OTKA grant N 25487 of The Netherlands and Hungary, the FAPESP grants 99/09113-3 00/04422-7 and 02/11344-8 of São Paulo, Brazil, and by the US DOE grants DE-FG02-93ER40764 and DEFG02-01ER41190. 


\section{References}

[1] T. Csörgö, F. Grassi, Y. Hama and T. Kodama, Simple solutions of relativistic hydrodynamics for longitudinally expanding systems, hep-ph/0203204.

[2] R. C. Hwa, Phys. Rev. D10 (1974) 2260,

C. B. Chiu and K.-H. Wang, Phys. Rev. D12 (1975) 272,

C. B. Chiu, E. C. G. Sudarshan and K.-H. Wang, Phys. Rev. D12 (1975) 902

M. I. Gorenstein, V. I. Zhdanov and Yu. M. Sinyukov, Sov. Phys. JETP 47 (78) 435.

K. Kajantie and L. D. McLerran, Phys. Lett. B 119 (1982) 203.

K. Kajantie and L. D. McLerran, Nucl. Phys. B 214 (1983) 261.

J.D. Bjorken, Phys. Rev. D27 (1983) 140.

[3] T. Csörgő, F. Grassi, Y. Hama and T. Kodama, Simple solutions of relativistic hydrodynamics for cylindrically expanding systems, hep-ph/0204300.

[4] I.M. Khalatnikov, Zhur. Eksp. Teor. Fiz. 27 (1954) 529; see also [5].

[5] L.D. Landau, Izv. Akad. Nauk SSSR 17 (1953) 51; S.Z. Belenkij and L.D. Landau, Usp. Fiz. Nauk 56 (1955) 309; in "Collected papers of L.D. Landau" (ed. D. Ter-Haar, Pergamon, Oxford, 1965) p. 569-585 and p. 665-700.

[6] L.P. Csernai, Introduction to Relativistic Heavy Ion Collisions, John Wiley and Sons, 1994.

[7] T.S. Biró, Phys. Lett. B474 (2000) 21-26.

[8] T.S. Biró, Phys. Lett. B487 (2000) 133-139.

[9] C.E. Aguiar, T. Kodama, T. Osada, Y. Hama, J. Phys. G27 (2001) 75-94.

[10] C.E. Aguiar, Y. Hama, T. Kodama, T. Osada, Nucl. Phys. A698 (2002) 639642.

[11] J. Bondorf, S. Garpman and J. Zimányi, Nucl. Phys. A296 (1978) 320.

[12] J.N. De, S.I.A. Garpman, D. Sperber, J.P. Bondorf and J. Zimányi, Nucl. Phys. A305 (1978) 226.

[13] P. Csizmadia, T. Csörgő and B. Lukács, nucl-th/9805006, Phys. Lett. B443 (1998) 21.

[14] T. Csörgö, nucl-th/9809011.

[15] S.V. Akkelin, T. Csörgö, B. Lukács, Yu.M. Sinyukov and M. Weiner, Phys. Lett. B 505 (2001), 64.

[16] T. Csörgö, S.V. Akkelin, Y. Hama, B. Lukács and Yu.M. Sinyukov, hep-ph/0108067.

[17] T. Csörgö, hep-ph/0111139. 
[18] M. Gyulassy and T. Matsui, Phys. Rev. D29 (1984) 419

[19] P. Lévai and U. Heinz, Phys. Rev. C57 (1998) 916;

A. Peischer, B. Kämpfer, G. Soff, Phys. Rev. C61 (2000) 045203;

A. Peischer, B. Kämpfer, G. Soff, Phys. Rev. D66 (2002) 094003;

A. I. Tóth and K. K. Szabó, hep-ph/0302255.

[20] T. Csörgő and L. P. Csernai, Phys. Lett. B333 (1994) 494

[21] L. P. Csernai and I. N. Mishustin, Phys. Rev. Lett. 74 (1995) 5005

[22] F. Grassi, Y. Hama and T. Kodama, Phys. Lett. B355 (1995) 9, Z. Phys. C 73 (1996) 153.

[23] Yu. M. Sinyukov, S. V. Akkelin and Y. Hama, Phys. Rev. Lett. 89 (2002) 052301

[24] T. Csörgő and B. Lörstad, Phys. Rev. C54 (1996) 1390; T. Csörgő and B. Lörstad, Nucl. Phys. A590 (1995) 465c. 